\title{
Qualidade de vida em mulheres mastectomizadas: possibilidades de ação do enfermeiro
}

Maria Lúcia Finotti Cherézio*, Rita Maria da Silva Grossi*, Benedito Cherbéu Dlessandre Oliveira, M.Sc.**

*Enfermeiras, Instituição de Ensino São Francisco de Mogi Guaçu, **Enfermeiro, Professor do Centro Universitário de Espirito Santo do Pinhal, Instituição de Ensino São Francisco e Coordenador do Curso de Enfermagem da ETEC Pedro Ferreira Alves, filiada ao Centro Paula Souza

\section{Resumo}

O objetivo desta revisão da literatura foi levantar as possibilidades de ação do enfermeiro no que tange auxiliar as mulheres mastectomizadas na busca por melhoria de qualidade de vida. Utilizou-se de publicaçóes em periódicos e livros publicados a partir de janeiro de 2000. O câncer de mama é o mais prevalente entre as mulheres e, no Brasil, seu prognóstico é agravado pelo alto índice de detecção tardia, o que dificulta a obtenção de uma melhor qualidade de vida às mulheres acometidas por essa doença. A rede básica de saúde tem papel fundamental no ensino do auto-exame e detecção precoce do câncer de mama devendo explorar mais esse potencial, e o profissional enfermeiro, inserido nesse contexto, tem papel essencial no atendimento dessas mulheres. Observa-se a escassez do especialista em oncologia, o que facilitaria o reconhecimento de grupos de risco e uma melhor adesão dessas mulheres nos programas de saúde. Percebe-se também a importância da parceria com a rede familiar e social como estratégia no enfrentamento da doença e busca por qualidade de vida. Este estudo não encerra o tema, que ainda necessita de aprofundamento para que novas possibilidades de atenção possam ser elaboradas prestigiando uma maior adesão dos profissionais e das mulheres na detecção precoce do câncer de mama, facilitando uma melhor qualidade de vida às mesmas.

Palavras-chave: qualidade de vida, mastectomia, enfermagem.

\section{Abstract}

\section{Women's quality of life after mastectomy: possibilities of nurse's actions}

This literature review has as objective to find out possibilities of nurse actions to help women, after mastectomy, to have better quality of life. The study researched journals and books published from January 2000. Breast cancer is the most common cancer among women and, in Brazil, its prognosis is worsened due to high rate of cancer delay detection, which makes difficult to improve quality of life of these women. Basic Health has an important role in teaching and demonstrating breast selfexamination and early detection of breast cancer and this potential should be more explored. Nurse professional, inserted on 
this context, has an essential role providing care for these women. It was observed lack of this professional on oncology, which would be of great importance to recognize risk groups and to promote adhesion of these women to Health programs. Also, we feel the importance of social and family support as strategy to face the disease and to look for better quality of life. This study does not stop this theme, it needs more researches in order to build new possibilities of caring with more professionals and women's adhesion in order to early detect breast cancer, providing better quality of life for these women.

Key-words: quality of life, mastectomy, nursing.

\section{Resumen}

\section{Calidad de vida en mujeres mastectomizadas: posibilidades de acción del enfermero}

Este estudio consistió de una revisión bibliográfica que tuvo el objetivo de verificar las posibilidades de acción del enfermero en ayudar a mujeres mastectomizadas en la búsqueda por una mejor calidad de vida. Fueron utilizados periódicos y libros a partir del 2000. El cáncer de mama es el tumor más prevalente entre las mujeres y, en Brasil, su pronóstico se agrava por el alto índice por detección tardía, lo que dificulta la obtención de una mejor calidad de vida para esas mujeres acometidas por esa enfermedad. La zona básica de salud tiene papel fundamental en la enseńanza del autoexamen y detección precoz del cáncer de mama, explorando más ese potencial, y el profesional enfermero, dentro de este contexto, tiene papel central en la atención a esas mujeres. Se observa la escasez de especialistas en oncología, lo que facilitaría el reconocimiento de grupos de riesgo y una mayor adhesión de esas mujeres a los programas de salud. Se percibe también la importancia del apoyo familiar y social como estrategia para mantener una actitud de lucha contra esa enfermedad y la búsqueda por calidad de vida. Este estudio no agota el tema, que aún necesita profundizarse para que nuevas posibilidades de atención se puedan plantear prestigiando una mayor adhesión de profesionales y de mujeres en la detección precoz del cáncer de mama, facilitando una mejor calidad de vida para las mismas.

Palabras-clave: calidad de vida, mastectomía, enfermería.

\section{Introdução}

Considerando a importância do tema, buscouse aprofundar a discussão acerca das relaçôes entre qualidade de vida $(\mathrm{QV})$ de mulheres mastectomizadas e as possibilidades de ação do enfermeiro. Através de uma revisão sumária da literatura estabeleceu-se um diálogo interdisciplinar, na busca do avanço do conhecimento e da relevância de um tema considerado de grande importância, tanto para a prática hospitalar quanto para a saúde coletiva, na busca constante de melhoria na promoção da saúde.

O termo QV abrange significados diversos como conhecimentos, experiências e valores de indivíduos e coletividade refletindo o momento histórico, social e cultural ao qual pertencem. A pesquisa da QV em saúde é de valor imprescindível, já que contribui para a determinação de tratamentos e avaliaçáo de custo-benefício dos mesmos [1].

Muitos estudos são encontrados na literatura internacional que avaliam QV, que foi definida pelo World Health Organization Quality of Life (WHOQOL) como "a percepção do indivíduo de sua posiçáo na vida, no contexto cultural e do sistema de valores em que vive e em relação aos seus objetivos, expectativas, padrões e preocupações" [2].

Em 1994, na abertura do 20 Congresso de Epidemiologia, Rufino Netto refere que "QV boa ou excelente é aquela que oferece um mínimo de condiçóes para que os indivíduos possam desenvolver ao máximo suas potencialidades como: viver, sentir e amar, trabalhar, produzindo bens e serviços, fazendo ciência ou artes". Ressalta ainda que "falta o esforço de fazer da noção um conceito e torná-lo operativo" [3]. A classe médica utiliza o termo QV para designar o bom funcionamento dos sistemas do organismo, perdendo a noção dessa qualidade como algo holístico e que traz implicações sócio-culturais ao indivíduo.

A literatura nacional apresenta pesquisas que buscam mensurar QV e para isto utilizam questionários gerais e específicos já validados ou desenvolvidos para grupos específicos, porém, existe a necessidade da avaliação de vários aspectos para mensurar a QV, tais como: apresentação de sintomas e seu grau de acometimento, o estado emocional e de humor, a adaptação da aparência física. É destacada a utilização desses instrumentos, no entanto os instrumen- 
tos específicos apresentam-se mais sensíveis para doenças crônico-degenerativas, os mais eleitos para o uso clínico [4].

O profissional de enfermagem é o que mais tempo permanece junto ao cliente e seus familiares, sendo considerado um verdadeiro elo com todos os envolvidos, interando-os na busca por recursos que possibilitem proporcionar uma melhor QV à pessoa enferma [5]. Podemos então considerar que o enfermeiro age neste contexto como agente transformador e tradutor das informaçôes técnicas para o cotidiano do indivíduo, facilitando o entendimento dessas, e proporcionando a sensaçáo de tranqüilidade e QV para seus clientes.

$\mathrm{O}$ enfermeiro pode interagir com seu cliente como agente terapêutico no relacionamento interpessoal transformando situaçóes de cuidado em experiências de aprendizagem e crescimento recíproco [6].

Diversos são os tratamentos direcionados ao portador de câncer e os enfermeiros os reconhecem, porém, é necessário que as prioridades não recaiam apenas no manejo da doença, mas que haja entendimento de todo o ambiente construído ao seu redor. São infinitivamente complexas as funçóes que envolvem o senso do corpo, sendo essas a soma das suas particularidades biológicas, em especial, no câncer. Portanto, além das técnicas utilizadas no tratamento, o enfermeiro também deve incluir o mundo do corpo do paciente, no sentido sociológico [7].

O câncer é considerado uma doença crônicodegenerativa e pelo diagnóstico precoce e evolução técnico-científica conferem possibilidade de cura ou uma sobrevida maior ao seu portador [8]. O câncer de mama é considerado um grave problema de saúde pública, por conseqüência, é uma das mais importantes causas de morbimortalidade na mulher em quase todo o mundo, sendo assim, a detecção precoce possibilita um tratamento menos mutilante e cerca de setenta por cento dos cânceres de mama são tardiamente diagnosticados dificultando a possibilidade de cura. A cirurgia ainda é o processo mais utilizado para prevenir a disseminaçáo da doença, sendo que a técnica dependerá da gravidade do quadro [9].

Segundo estimativas do Instituto Nacional do Câncer [10], o número de casos novos de câncer de mama esperados para o Brasil em 2008 é de 49.400, com um risco estimado de 51 casos a cada $100 \mathrm{mil}$ mulheres.

A situação vivenciada pela mulher mastectomizada imprime à equipe de saúde reflexáo acerca do papel dos profissionais no atendimento à mulher, isto porque, as mamas, símbolos de beleza, sensualidade e fonte de alimento aos recém-natos, quando estirpadas cirurgicamente, gera na mulher variados sentimentos. $\mathrm{O}$ vazio configurado na percepção da mulher associa-se ao medo da doença, a desconfiguração do "normal" e a problemas sexuais com o parceiro. Todas estas apreensóes percebidas pela mulher mastectomizada podem ser superficialmente sentidas pela enfermagem. Vale ressaltar que, a enfermagem deve subsidiar a assistência com um ouvir diferenciado, fornecendo o aporte emocional necessário à cliente.

Entende-se como objetivo desse trabalho, discutir as possibilidades de ação do enfermeiro no auxílio à busca por QV das mulheres mastectomizadas.

\section{Material e método}

Foi realizado estudo de revisão bibliográfica sobre a temática "qualidade de vida de mulheres mastectomizadas", sendo que os dados obtidos foram selecionados através de livros e artigos científicos, quando esses se mostraram relevantes à temática, no período de janeiro de 2000 a março de 2007. Foram usados os descritores: enfermagem, qualidade de vida, oncologia, câncer de mama e mastectomia.

\section{Consideraçóes acerca do câncer de mama}

O câncer de mama é mais comum em mulheres pós-menopausa, e o Brasil carrega em suas estatísticas ser o país que apresenta o maior número de casos, tendo também o pior diagnóstico entre as mulheres jovens em relação às idosas [11].

Os fatores de risco para o câncer de mama, segundo o Ministério da Saúde, abrangem: menarca precoce, menopausa tardia, primeira gestação após os trinta anos, nuliparidade, doenças mamárias, história familiar de câncer de mama e dieta rica em gorduras. O controle do câncer de mama é preconizado pelo Ministério da Saúde, porém, não raramente as mulheres são desestimuladas pelo difícil acesso e demora no atendimento nos serviços de saúde, agravados pela falta de profissionais qualificados [12].

É reconhecida a multiplicidade do câncer, sendo que o de mama traz uma alteração singular às suas portadoras, levando essas a questionamentos como mulher. E por parte dos profissionais de saúde 
há a necessidade do desenvolvimento de habilidades que auxiliem essas mulheres no enfrentamento das dificuldades do tratamento e suas conseqüências [13].

Não raramente existem as dificuldades da rede básica de saúde na detecçáo precoce do câncer de mama acrescentados aos fatores que diz respeito às mulheres, tais como, seus preconceitos e timidez na realizaçáo do auto-exame, elementos esses que podem interferir de forma singular na detecção precoce [14]. O câncer ainda é uma das doenças que mais causa incertezas ao seu portador em qualquer estágio, começando pelo impacto do diagnóstico, incertezas quanto aos tratamentos e seus resultados, medo do prognóstico, sofrimento e possível morte [15].

A complexidade do tratamento da pessoa com câncer, por vezes, acaba debilitando o organismo como um todo. Este processo de mudança pode acabar interferindo negativamente também na autoimagem corporal comprometendo a sexualidade humana deste indivíduo, afetando seu cotidiano nos aspectos biopsicossocial podendo evoluir para quadros depressivos [16].

O diagnóstico do câncer, entre eles o de mama, repercute profundamente no cotidiano dessas mulheres, ocasionado pelas mudanças no modo de viver devido ao tratamento, podendo interferir, muitas vezes, de forma negativa na QV necessitando de intervençóes para amenizar esse impacto [17]. Com a evolução dos tratamentos oncológicos tem ocorrido uma maior sobrevida dessas pacientes, necessitando de recursos, em especial humanos, que auxiliem na busca de uma melhor QV para as envolvidas [18].

O diagnóstico de câncer normalmente é recebido com impacto, muitas vezes negativo na vida do portador, levando-o a questionamentos mútuos. Por outro lado, pode ser considerado como um divisor de águas, onde a pessoa passa a repensar seu modo de vida e, a partir daí, resgata valores e princípios, muitas vezes esquecidos por interesses materialistas [19].

Diante do resultado observado na literatura pesquisada, tornou-se evidente a necessidade da efetividade dos profissionais de saúde envolvidos com essa clientela. $\mathrm{O}$ enfermeiro deve desenvolver seu papel tanto de cuidador quanto de educador em saúde, possibilitando uma maior adesão das mulheres no auto-exame, facilitando o diagnóstico precoce, possibilitando maior chance de cura ou mesmo uma maior sobrevida às mulheres acometidas pelo câncer de mama.

\section{A mastectomia e suas representaçóes}

A mastectomia consiste na retirada da glândula mamária por meio de terapia cirúrgica. $\mathrm{O}$ tipo de cirurgia vai depender do estágio clínico da doença, de achados radiológicos, localização do tumor, história da cliente, experiência cirúrgica e em radioterapia disponível, tamanho e formato da mama e preferência da cliente [20].

O grau de percepção da mulher no que tange o impacto da mastectomia em seu cotidiano irá determinar o controle no auto-exame e a procura por profissionais de saúde [21].

Pela representação física e emocional que a mastectomia acarreta às mulheres, elas experienciam o impacto da mutilação, da limitação física, da necessidade de cuidado com seu próprio corpo, da sensaçáo de impotência ao retornar para casa, e, por fim, da necessidade de conformar-se e adaptar-se a essa nova condiçáo [22].

O desajuste por que passam as mulheres mastectomizadas pode levar a traumas psicológicos pela impossibilidade do retorno normal às suas atividades de vida diária logo após a cirurgia. Por outro lado, o positivismo da convivência e troca de experiências com mulheres que já vivenciaram a mastectomia, possibilita a diminuição dos estigmas da doença e uma maior interaçáo social [15].

A relação familiar saudável torna possível um melhor enfrentamento da doença e, o parceiro sexual tem um papel fundamental nesse processo, sendo estimulador e cuidador nessa nova fase de reabilitação [23]. Observa-se também nas mulheres mastectomizada, a substituição da imagem corporal perfeita por uma imagem distorcida, levando-as a sentirem-se rejeitadas na sociedade, predispondo-as ao isolamento social, e a realidade de uma doença grave que pode ser revertida em estratégias de enfrentamento, levando-as a repensar seu cuidado com a saúde [19].

Diversos são os tratamentos direcionados à mulher mastectomizada. Estes incluem entre outros a quimioterapia, a radioterapia e os tratamentos hormonais. Neste capítulo o levantamento bibliográfico realizado nos leva a concluir que a mulher mastectomizada necessita de um tratamento individualizado. Ao enfermeiro, cabe o desenvolvimento da percepção acerca das necessidades da mulher para a elaboração de um plano de cuidados que facilitem a busca destas por uma melhor QV. 


\section{O que vem a ser qualidade de vida?}

O termo QV, em especial na área da saúde, está intimamente relacionado às condiçóes de vida de uma população, normalmente mensurada à oferta de infra-estrutura básica, educação, trabalho, habitação, saúde entre outras necessidades peculiares a cada grupo sociocultural distinto.

Diferentes autores e relatórios sobre saúde mundial, World Health Organization (WHO, 1998) e da regiáo das Américas, Organização PanAmericana da Saúde (OPAS, 1998), salientam que as condiçóes de vida têm melhorado graças aos progressos políticos, econômicos, sociais e ambientais elevando consideravelmente a expectativa de vida dos indivíduos. Também, a desigualdade social, onde problemas de saúde já sanados em muitos lugares e persistentes em outros, como é o caso das doenças parasitárias, alertam para as novas questóes que emergem como problemas de saúde na atualidade (drogas, violência, estresse), necessitando de desenvolvimento de estratégias para o enfrentamento das mesmas [24].

O grupo de estudo sobre QV da Organização Mundial da Saúde (OMS) define QV como "a percepção do indivíduo de sua posição na vida, no contexto da cultura e sistemas de valores nos quais vive e em relação aos seus objetivos, expectativas, padrões e preocupações", são seis os domínios principais que abrangem esta definição: "saúde física, estado psicológico, níveis de independência, relacionamento social, características ambientais e padrão espiritual" [1].

A definição da promoção da saúde descrita na carta de Ottawa (WHO, 1986) se dá da seguinte forma: "o processo de capacitação da comunidade para atuar na melhoria da sua qualidade de vida e saúde, incluindo uma maior participação no controle deste processo". No Brasil pode-se observar esse processo por meio da mudança de estratégia que vem sendo implantada com a introdução dos Programas de Agentes Comunitários de Saúde e Saúde da Família, amparados ainda pela mudança legislativa e melhoria na renda, impulsionando desta forma a QV e condiçóes de saúde da população [24].

A tendência universal, incluindo os países em desenvolvimento, está no aumento da prevalência das doenças crônico-degenerativas e do perfil de morbimortalidade, sendo a oncologia, a especialidade que mais tem se preocupado com a mensuração da QV de seus portadores devido aos avanços tecnológicos e efetividade dos tratamentos. A QV inicialmente era avaliada por um observador, método hoje já contestado por vários estudiosos, que afirmam que a $\mathrm{QV}$ só pode ser avaliada pela própria pessoa [25].

A QV abrange aspectos relacionados “... ao grau de satisfaçáo encontrada na vida familiar, amorosa, social e ambiental e à própria estética existencial", pode ser determinada pelos valores culturais e sociais variando individualmente conforme os graus de expectativas e a realidade vivenciada [3].

É observada a importância do papel da família no cotidiano de mulheres com câncer, onde a busca por saúde e QV é compartilhada, muitas vezes, pela troca dos mais diversos sentimentos humanos como "afetividade, carinho e compreensão" [26].

Baseado na literatura encontrada, infere-se que o enfermeiro deve se inserir neste contexto como agente facilitador desse processo, onde, a partir de conhecimentos teóricos enriquecidos pela sua vivência prática, ajuda a cliente a buscar caminhos que facilitem o enfrentamento da doença e a busca por uma melhor qualidade de vida.

Quais as possibilidades de atuação do enfermeiro no auxílio ao resgate da qualidade de vida em mulheres mastectomizadas?

$\mathrm{Na}$ área da saúde, a enfermagem se destaca por ser a profissáo que mais tempo permanece junto ao cliente, e esse cuidado abrange todos os níveis de prestação de serviços, que vai desde a promoção da saúde, a prevenção de doenças, o tratamento em todas as fases da patologia até a reabilitaçáo, quando essa se faz necessário. Diante dessa premissa, o enfermeiro deve agir como educador e facilitador nas oportunidades que ocorrem na relação enfermeiro/ cliente, criando possibilidades de aprendizagem mútua e facilitando a busca por uma melhor QV de seus clientes.

$\mathrm{O}$ cuidado de enfermagem tem que atingir as totais necessidades do ser humano, e, na atualidade, percebe-se que esse cuidado vem se tornando mecanicista, dificultando assim a obtenção de resultados positivos. Cabe ao enfermeiro reconhecer seu próprio "eu" para depois auxiliar o outro na sua existência [27].

Os enfermeiros deveriam explorar mais seu campo de atuação no que tange a conscientização e esclarecimentos sobre o autocuidado e diagnóstico precoce do câncer de mama, possibilitando à mulher uma maior adesão aos programas de saúde e seus direitos como cidadá [11]. 
As mulheres, no seu existir vão acumulando experiências que refletirão em suas escolhas futuras, e isto é evidenciado na percepção do câncer de mama implicando no autocuidado e busca precoce de tratamento, e ao enfermeiro cabe assistir efetivamente essas mulheres, realizando a abordagem conforme o grau de conhecimento de cada uma, no intuído de atingir de forma adequada tanto a assistência quanto à educaçáo em saúde [21]. Nesse contexto o enfermeiro deve desenvolver estratégias que facilitem o esclarecimento da importância do auto-exame, do diagnóstico precoce e dos variados modos de tratamento do câncer de mama.

Existe a importância do papel do enfermeiro como educador no processo de aceitação da doença e reabilitação frente a grupos de auto-ajuda, onde a cliente deve ser vista na sua integralidade e individualidade, sendo que o tratamento humanizado se perfaz por um profissional presente e atencioso, características que não devem faltar ao enfermeiro, em especial, na área da oncologia [15].

É observada uma crítica à falta de profissionais preparados para o atendimento de mulheres portadoras de câncer de mama, e o aprimoramento do enfermeiro facilitaria o feedback necessário na interação enfermeiro/cliente, diminuindo assim os riscos de complicaçóes pós-operatórios e facilitando as articulaçóes com outros profissionais envolvidos no atendimento dessa clientela [28].

Uma das ferramentas que o enfermeiro possui está na comunicação, que deve acontecer de forma harmoniosa, possibilitando a criação de um vínculo de confiança entre enfermeiro/cliente. Podemos afirmar, ainda, que a comunicação proporciona maior possibilidade de tratamento eficaz e humanizado. A assistência integrada e contínua do enfermeiro no período de internação proporciona uma maior adesão ao tratamento e autocuidado por parte das clientes que percebem suas reais necessidades alcançadas, garantindo assim o envolvimento necessário enfermeiro/cliente [29].

Outra forma de cuidado é a comunicação verbal e a não-verbal e o enfermeiro deve desenvolver esta habilidade, facilitando desta forma a identificaçáo dos problemas e a busca por soluçóes [30].

Observa-se a importância do elo enfermeiro/ cliente/rede social no processo de reabilitação da mulher mastectomizada, onde o parceiro se destaca como aliado do enfermeiro para que as metas sejam alcançadas [23].
A tecnologia tem modificado o prognóstico oncológico e, diante desse fato, há a necessidade do aprimoramento do enfermeiro tanto técnico quanto emocional, o que facilitaria o reconhecimento de reaçóes observadas no período de adaptação e ajuste psicológico da cliente aumentando a probabilidade da eficácia do tratamento [8].

A comunicação efetiva enfermeiro/cliente facilitará a busca por soluções de problemas no percurso do tratamento, além disso, proporcionará condiçôes para a elaboraçáo de novos padróes de comportamento por parte da cliente, os quais auxiliaráo na melhoria de sua QV [31].

A reflexáo bioética deve fazer parte do cotidiano do enfermeiro na sua relaçáo com a mulher acometida por câncer de mama, facilitando a busca destas por autonomia sobre seu corpo e seu tratamento [32].

$\mathrm{O}$ enfermeiro envolvido com pacientes oncológicos náo deve se ater apenas aos cuidados biológicos, mas procurar compreender sua doença e as suas conseqüências, elaborando um plano de cuidados que contemple o bem estar físico e emocional desta cliente [7].

Durante a evoluçáo do tratamento do paciente oncológico, vai se instalando uma relação de troca mútua enfermeiro/cliente, que vai além do cuidado físico, envolve afetividade. O enfermeiro deve desenvolver estratégias para lidar com as diversas situações que poderão surgir, entre elas a morte, que, por vezes, pode gerar conflitos existenciais e interferir de forma negativa na vida desse profissional [33].

Pelas leituras realizadas, os autores reconhecem que ao enfermeiro cabe agir neste contexto com pensamento crítico, proporcionando um cuidado individualizado, adequando o ambiente às necessidades, interagindo nas relaçóes interpessoais de forma harmoniosa, gerenciando e administrando situaçóes que favoreçam o restabelecimento adequado da cliente.

\section{Percepçóes acerca do cuidar em oncologia}

Diante da incidência do câncer de mama feminino e da evoluçáo dos tratamentos que tem permitido uma maior chance de cura e sobrevida, é pertinente a preocupação do enfermeiro na mensuração da QV desse grupo, bem como a discussão acerca das possibilidades de ação no seu cotidiano, favorecendo assim uma assistência que atinja e respeite a individualidade de cada cliente. 
A rede básica de saúde necessita de estratégias que facilitem à mulher reconhecer-se capaz de se auto-examinar, o que possibilitaria a detecção precoce do câncer de mama [14]. No Brasil existe uma maior preocupaçáo em mensurar as causas da mortalidade feminina em vez de desenvolver estratégias que interfiram na diminuição destas, incluindo o índice de câncer de mama. Além disso, há pouca oferta de profissionais qualificados na área oncológica [12].

Percebe-se a necessidade de profissionais com habilidades específicas para auxiliar a mulher no enfrentamento do câncer de mama [13]. Também é observado a não procura pelo diagnóstico precoce por parte da mulher, tendo como barreira o fato de já ter vivenciado a doença na família [7].

Alguns autores referem a relevância na terapia de grupos e da participaçáo das famílias e amigos na evoluçáo do tratamento e reafirmam isto quando enfatizam que a mulher necessita de ajuda durante o percurso da doença $[5,11]$. Sabemos que a mastectomia não encerra o tratamento do câncer de mama, em especial quando esse procede de radioterapia sendo mais comuns as complicaçóes e limitaçóes, e percebe-se que a cirurgia conservadora favorece uma maior QV à mulher [21].

A decisão pela mastectomia acaba sendo do médico. Nesse momento, a mulher está com suas preocupaçóes voltadas ao tratamento e a uma possível recorrência da doença, e uma cirurgia conservadora possibilitaria uma maior QV, porém, a mulher acaba se colocando em condiçóes desfavoráveis para decidir o modo de tratamento, provavelmente pelos fortes estigmas da doença que traz enraizadas incertezas quanto ao futuro [24].

Várias linhas de pensamento têm definido $\mathrm{QV}$, porém todos concordam que é uma percepção individual que sofre fortes influências de fatores externos [3,24,25]. Na área clínica percebe-se que a mensuração da QV prestigia a avaliação dos tratamentos e custo/benefícios dos mesmos, nem sempre respeitando a individualidade de cada um [1].

É reconhecida a dimensão do campo de atuação do enfermeiro que abrange todos os estágios do cuidar, que vão desde a promoçáo, a prevençáo, o tratamento e a reabilitaçáo da cliente.

São apontadas várias formas de atuar que contribuam com o cuidar dessas clientes, dentre elas podemos citar: empatia, comunicação verbal e nãoverbal, assistência integrada e contínua, envolvimento com grupos de apoio destacando a importância da família e do parceiro, aprimoramento técnico e emocional, reflexão bioética, orientação e educação no período pós-operatório, e por fim, a importância do envolvimento em pesquisa oncológica direcionada à enfermagem $[8,22,23,26,29.30,32]$.

Após o levantamento realizado existe a concordância com os autores nos achados, e acrescenta-se que há a necessidade de mais estudos para direcionar caminhos que se possam trabalhar com essas clientes, possibilitando uma maior adesão ao autocuidado e a detecção precoce, fatores essenciais na facilitação da busca por uma melhor QV dessas mulheres.

\section{Conclusão}

Na pesquisa constatou-se que o auto-exame das mamas e diagnóstico precoce do câncer de mama são fatores essenciais para proporcionar condiçóes de uma melhor QV às mulheres que necessitam submeter-se à mastectomia. Observamos também a importância na atuação da rede básica de saúde no que tange a esses cuidados, e a necessidade da elaboração de estratégias que facilitem a detecçáo precoce do câncer de mama, possibilitando a diminuição dos diagnósticos tardios, ainda prevalentes no Brasil.

Percebeu-se ainda o papel imprescindível do enfermeiro como elo entre todos os envolvidos no cotidiano das mulheres mastectomizadas, porém, é reconhecida a escassez do enfermeiro especializado em oncologia, em especial, no atendimento a essas mulheres.

A identificação pelo enfermeiro de grupos que apresentam fatores de risco para o câncer de mama, preconceitos quanto ao auto-exame, ausência ou baixa percepção do impacto da mastectomia irão determinar estratégias na abordagem dessas clientes, para que possam desenvolver seu potencial de autocuidado e procura por profissionais de saúde, propiciando uma melhor QV.

$\mathrm{O}$ enfermeiro deve estabelecer suas ações de forma que proporcione um cuidado diferenciado, sendo agente educador e facilitador nas açóes de prevenção, desenvolvendo planejamento individualizado no percurso do tratamento, criando vínculos com os envolvidos, possibilitando uma melhor adesão ao tratamento e participação em grupos de auto-ajuda, valorizando o papel da família, amigos e, principalmente, do parceiro no enfrentamento e adaptação da mulher nessa nova fase.

Em qualquer estágio que esteja o cuidar, o enfermeiro deve agir com primazia, elemento faci- 
litador para a busca de uma melhor QV para essas clientes.

Esse trabalho não esgota o tema, há a necessidade de aprofundamento e publicações de estudos que prestigiem a $\mathrm{QV}$ de mulheres mastectomizadas. Recomenda-se aos enfermeiros especializados em oncologia que direcionem seus estudos para a facilitação da QV em mulheres mastectomizadas e as possibilidades de ação do enfermeiro, prestigiando assim a melhoria da saúde da mulher.

\section{Referências}

1. Dantas RAS, Sawada NO, Malerbo MB. Pesquisas sobre qualidade de vida: revisão da produçáo científica das Universidades Públicas do Estado de São Paulo. Rev Latinoam Enfermagem 2003;11(4):532-8.

2. Toledo RCMR. Qualidade de vida: adaptaçáo cultural do Spitzer Quality of Life Index. [Dissertação]. Campinas: Faculdade de Ciências Médicas, Universidade Estadual de Campinas; 2006.

3. Minayo MCS, Hartz ZMA, Buss PM. Qualidade de vida e saúde: um debate necessário. Ciênc Saúde Coletiva 2000;5(1):7-18.

4. Moreira ECH, Manaia CAR. Qualidade de vida das pacientes mastectomizadas atendidas pelo serviço de fisioterapia do Hospital Universitário da Universidade Estadual de Londrina. Semina Cienc Biol Saúde 2005;26(1):21-30.

5. Carvalho MVB, Merighi MAB. O cuidar no processo de morrer na percepçâo de mulheres com câncer: uma atitude fenomenológica. Rev Latinoam Enfermagem 2005;13(6):951-9.

6. George JB. Teorias de Enfermagem: os fundamentos a pratica profissional. 4a ed. Porto Alegre: Artes Médicas Sul; 2000. p. 45-57.

7. Anjos ACY, Zago MMF. A experiência da terapêutica quimioterápica oncológica na visão do paciente. Rev Latinoam Enfermagem 2006;14(1):33-40.

8. Silva MAPD, Silva ARB, Silva EM. Oncologia e ética: relaçóes e aproximaçóes. Rev Paul Enfermagem 2001;20(1):42-50.

9. Granja CF. O impacto físico-emocional do câncer de mama em mulheres submetidas a tratamento cirúrgico: uma abordagem fisioterapêutica. [TCC]. Cascavel: Unioeste; 2004.

10. Brasil. Ministério da Saúde. Instituto Nacional do Câncer. Estimativa 2008 Brasil Consolidado [online]. [citado 2009 mai 26]. Disponível em URL: http://www.inca.gov.br/estimativa/2008/index. asp?link=conteudo_view.asp\&ID $=5$

11. Araújo JS, Nascimento MAA. Atençáo da família frente ao processo saúde-doença de um familiar com câncer de mama. Rev Bras Enfermagem 2004;57(3):274-8.

12. Fialho AVM, Silva RM. Câncer de mama: o pensar e o fazer das mulheres. Rev Bras Enfermagem 2004;57(2):157-60.

13. Cantinelli FS, Camacho RS, Smaletz O, Gonsales BK, Braguittoni E, Rennó Junior J. A oncopsiquiatria no câncer de mama - consideraçôes a respeito de questôes do feminino. Rev Psiquiatr Clín (São Paulo) 2006;33(3):124-33.
14. Davim RMB, Torres GV, Cabral MLN, Lima VM, Souza MA. Auto-exame de mama: conhecimento de usuárias atendidas no ambulatório de uma maternidade escola. Rev Latinoam Enfermagem 2003;11(1):21-7.

15. Fernandes AFC, Rodrigues MSP, Cavalcanti PP. Comportamento da mulher mastectomizada frente às atividades grupais. Rev Bras Enfermagem 2004;57(1):31-4.

16. Melo AS, Carvalho EC, Pelá NTR. The sexuality of patients with onco-hematological diseases. Rev Latinoam Enfermagem 2006;14(2):227-32.

17. Furlani R, Ceolim MF. Qualidade do sono de mulheres portadoras de câncer ginecológico e mamário. Rev Latinoam Enfermagem 2006;14(6):872-8.

18. Silveira CS, Zago MMF. Pesquisa brasileira em enfermagem oncológica: uma revisão integrativa. Rev Latinoam Enfermagem 2006;14(4):614-9.

19. Rzeznik C, Dall'Agnol, CM. (Re) Descobrindo a vida apesar do câncer. Rev Gaúch Enferm 2000;21(Supl):84100.

20. Otto SE. Câncer Mamário. Oncologia. Traduzido por: Gomes IL, Santos AB. Rio de Janeiro: Reichmann \& Affonso; 2002. p. 105-21.

21. Fugita RMI, Gualda DMR. A causalidade do câncer de mama à luz do modelo de crenças de saúde. Rev Esc de Enferm USP 2006;40(4):504-6.

22. Ferreira MLJM, Mamede MV. Representação do corpo na relaçâo consigo mesma após mastectomia. Rev Latinoam Enfermagem 2003;11(3):299-304.

23. Biffi RG, Mamede MV. Suporte social na reabilitação da mulher mastectomizada: o papel do parceiro sexual. Rev Esc Enferm USP 2004;38(3):262-9.

24. Buss PM. Promoçáo da saúde e qualidade de vida. Ciênc Saúde Coletiva 2000;5(1):163-77.

25. Seidl EMF, Zannon CMLC. Qualidade de vida e saúde: aspectos conceituais e metodológicos. Cad Saúde Pública 2004;20(2):580-8.

26. Sales CA, Molina MAS. O significado do câncer no cotidiano de mulheres em tratamento quimioterápico. Rev Bras Enfermagem 2004;57(6):720-3.

27. George JB,eds. Teorias de Enfermagem. 4a. ed. Porto Alegre: Artes Médicas Sul; 2000. p. 253-65.

28. Panobianco MS, Mamede MV. Complicaçóes associadas ao edema de braço nos três primeiros meses após mastectomia. Rev Latinoam Enfermagem 2002;10(4):544-50.

29. Sousa RA, Pessoa SMF, Herculano MMS, Vale MAP. A comunicação durante a visita ao leito como fator de qualidade da assistência de enfermagem. In: Anais do 8. Simpósio Brasileiro de Comunicação em Enfermagem [Anais online]; 2002 Maio 02-03; Sáo Paulo, SP, Brasil. 2002 [citado 2007 Mar 25]. Disponível em URL: http://www.proceedings.scielo.br/scielo.

30. Bobroff MC, Furegato ARF, Scatena MCM. O enfermeiro na relação de ajuda a paciente com câncer. Rev Paul Enfermagem 2003;22(3):158-65.

31. Silva MJP. Comunicação tem remédio: a comunicaçáa nas relações interpessoais em saúde. 4a ed. Sáo Paulo: Loyola; 2006.

32. Arantes SL, Mamede MV. A participação das mulheres com câncer de mama na escolha do tratamento: um direito a ser conquistado. Rev Latinoam Enfermagem 2003;11(1):49-58.

33. Popim RC, Boemer MR. Cuidar em oncologia na perspectiva de Alfred Schütz. Rev Latinoam Enfermagem 2005;13(5):677-85. 